\title{
Customized Software and Hardware applied to Assessment of Outwardly Spherical Flames Using the Pressure Trace: a Thermodynamic Approach to Improve Accuracy of Laminar Flame Speed Measurements
}

\author{
R. M. Hartmann*1, E. J. de Oliveira ${ }^{2}$, M. I. Rocha ${ }^{2}$, A. A. M. de Oliveira Jr. ${ }^{1}$ \\ ${ }^{1}$ Federal University of Santa Catarina, Florianópolis, Brazil \\ ${ }^{2}$ Petrobras R\&D Center, Rio de Janeiro, Brazil \\ E-mail: ricardo@labcet.ufsc.br
}

Received 15 December 2016, Revised 16 February 2017, Accepted 28 February 2017

\begin{abstract}
Outwardly propagating spherical flames are well suitable for measurements of Laminar Flame Speed which is a very important thermochemical property of flammable mixtures. There are several facilities, approaches and methods applied to measure Laminar Flame Speed. Due this variety of measurements, consistency and accuracy of the results are mandatory for useful comparisons. Confined premixed flames were assessed using a customized spherical stainless steel vessel, equiped with a Schlieren setup and a dynamic pressure transducer, allowing the comparison between the syncronized results obtained using both approaches. The thermodynamic method presented in this paper uses experimental pressure traces as input to a FORTRAN code that calculates temporal profiles of flame radius, flame surface area, burning velocity, the scalar laminar flame speed and some additional quantities. The proposed algorithm processes the raw pressure trace, controls flow data to an external code to calculate chemical equilibrium and thermodynamic properties and calculates the rate of reactants burned by the flame propagation. The strategy allows to obtain the laminar flame speed without the need to model heat transfer from the flame, diminishing uncertainties in the calculations. The calculations of the proposed FORTRAN code enables to obtain the Laminar Flame Speed at initial datum and the flame behavior for higher pressures and temperatures, maximizing the results for each experiment. Measurements were carried out for air mixtures with methane, iso-octane, $\mathrm{n}$-heptane, Jet A and Jet A-1. The laminar flame speed is the highest for Jet fuels at $\mathrm{T}_{\mathrm{i}}=408 \mathrm{~K}$ and lowest for iso-octane at $\mathrm{T}_{\mathrm{i}}=300 \mathrm{~K}$, about $50 \%$ higher for stoichiometric mixtures. The faster flames for Jet fuels presented the highest exergy destruction, about $30 \%$. The joint analyses of the results of flame radius, Laminar Flame Speed, exergetic assessment of the flame propagation and the calculated equilibrium composition of the burned gases showed the consistency and good accuracy of the thermodynamic approach here presented.
\end{abstract}

Keywords: Premixed flame propagation; exergy; jet fuels; gasoline PRF fuels; combustion.

\section{Introduction}

Equivalence and accuracy are key characteristics needed to allow useful comparisons among different measurements of Laminar Flame Speed (LFS). The LFS is defined as the relative velocity of reactants which cross the control volume of a laminar flame that is planar, adiabatic and stretch-free [1-4]. As the LFS is measured indirectly, using several different facilities, approaches and methods, the first chalenge is to compare the variety of results in a common frame, which should be physically meaningful and provide straightforward comparisons.

LFS has been measured since the beginning of the $20^{\text {th }}$ century using experimental sets like flat burners, Bunsen burners, opposite jet flows and constant volume reactors (CVR). It is important to note that the results always presented a high degree of scattering. Concerning this scattery behavior, Law and $\mathrm{Wu}$ [5] first introduced the concept of flame stretch rate, which was the first method to compare results of LFS in a common physical frame. Figure 1 shows the positive effect of the introduction of the concept of flame stretch rate. After the introduction of the concept of flame stretch rate, in the beginning of the 1980's, the scatter in LFS results has remarkably diminished. The utilization of the concept of flame stretch rate strongly contibuted to organize and to confirm the usefulness of the LFS results obtained using distinct facilities. After this first generalization, additional improvements were investigated, focusing on other aspects of mixture and flame propagation.

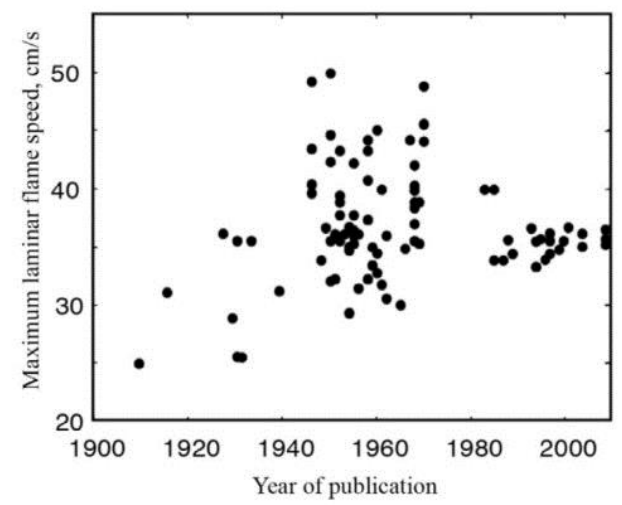

Figure 1. Compilation of results of laminar flame speed of stoichyomteric mixtures of methane and air obtained during the 20th century. Adapted from [6].

In the ensuing sections those aspects will be addressed and the results obtained in this work that led to further improvements will be shown. The specific improvements due to the CVR here used, due to the thermodynamic approach and due to the FORTRAN code will be listed. Foremost, a short review of LFS measurements will be 
presented, followed by a description of materials and methods employed. The results of flame radius, LFS and exergetic assessment of the flame propagation are used to validate the proposed method.

\section{Literature Review}

\subsection{Review of LFS Measurements Using CVR}

The interest to measure LFS started to increase about a century ago. Hopkins [8] was one of the first who reported results of premixed flame propagation using a CVR. Hopkins reported results of mixtures between air and coalgas. The averaged burning velocity was infered using a thermocouple and a pressure probe mounted at the wall of the reactor. Zabetakis [9] presented a comprehensive basis of burning rates of gaseous mixtures. Zabetakis carried out experiments using a cylindrical CVR with $19.7 \mathrm{~cm}$ diameter and 9 liter inner volume. Experiments for central ignited mixture between air with hydrocarbons and alcohols were reported. In a typical experiment, after a slow pressure increase during the first few seconds, the pressure trace presented a rapid increase until the flame reaches the walls of the reactor. Zabetakis utilized a simple thermodynamic model to correlate the pressure increasing and the flame propagation,

$$
\Delta p=K p_{i} \frac{\mathrm{V}_{B}^{3} t^{3}}{V}
$$

where $\Delta p$ is the pressure increase during the combustion, $\mathrm{K}$ is a proportionality constant, $p_{i}$ is the initial pressure, $V_{B}$ is the burning velocity, $t$ is the time and $V$ is the vessel volume. The method employed by Zabetakis was still an averaged method, where the burning velocity was evaluated using the pressure measured at the end of the flame propagation.

Metghalchi and Keck [10] presented a more sophisticated model to calculate the burning velocity that takes into account its variation during the outwardly flame propagation,

$$
\begin{aligned}
& V_{B}=\frac{m_{i}}{\rho_{u} A_{f l}} \frac{d y}{d t} \\
& V_{B}=\frac{\rho_{i}}{\rho_{u}}\left(\frac{V_{C}}{V_{f}}\right)^{2 / 3} \frac{r_{C}}{3}\left(\frac{d y}{d p}\right) \frac{d p}{d t}
\end{aligned}
$$

where $m_{i}$ is the initial mass of the mixture inside of the reactor, $y$ is the mass fraction of burned gases, $\rho_{u}$ is the unburned gas density, $A_{f l}$ is the flame surface area evaluated from the reactants side, $t$ is time, $\rho_{i}$ is the initial density, $V_{C}$ is the inner chamber volume, $V_{f}$ is the volume occupied by the burned gases, $r_{C}$ is the radius of the vessel and $V_{B}$ is the burning velocity. Eq. (2) is feeded by mass fraction obtained in the solution of an equation of conservation of specific volume within the reator. The evaluation of the volume equation is based on ideal gas hypothesis, the flame is spherical and smooth, the pressure is function only of time and both reactants and products of combustion are in chemical equilibrium. The main insight of the approach was to consider each step of the flame propagation as an isoenthalpic-isobaric process. One drawback is the need to obtain accurate functions $d y / d p$ and $d p / d t$ from the measurements.

Rahim et al. [11] presented an updated thermodynamic approach, where the necessity of analytical solutions was avoided. The model evaluates both conservation of volume and energy,

$E_{i}-Q_{l s}=E_{b}+E_{u}$

$V_{i}-V_{B L}=V_{b}+V_{u}$

where $E_{i}$ is the initial energy of the reactants, the term $Q_{l s}$ embodies all the flame energy transfer and dissipations: the heat transfer to the ignition electrodes, to the reactor walls through convection, radiation and conduction. $E_{b}$ and $E_{u}$ are the energy of the burned and unburned gases respectively. The same nomencalture scheme holds for the volume conservation Eq. (4), where $V_{i}$ is the volume actually ocupied by the reactants and the term $V_{B L}$ is related to the volume of boundary layers attached to the electrodes, reactor walls and to the flame front.

Using the experimental pressure profile as input in addition to the ideal gas equation and considering isentropic compression of reactants and burned gases, the system of equations can be solved using a Newton-Raphson algorithm, taking advantage of the capacity of modern computers. The solution of (3) and (4) will provide flame temperature and burned mass fraction as function of time. The burning velocity can thus readily be calculated similarly as Eq. (2)

$V_{B}=\frac{\dot{m}_{b}}{\rho_{u} A_{f l}}=\frac{m_{i} \dot{y}}{\rho_{u} A_{f l}}$

where $V_{B}$ is the burning velocity as function of time of the flame propagation. It is important to note that Eq. (5) yields the instantaneous value of burning velocity and is influenced by effects of flame stretch. Thus, it can also be called as stretched burning velocity.

\subsubsection{Flame Stretch Rate and Calculation of the Laminar Flame Speed}

Flames observed in combustion devices and in laboratory experiments are seldom perfectly planar. Occurrence of flows may induce flame instabilities and the flame propagation itself induces instabilities, intrinsically related to hot gases' expansion [12]. This is the case of propagation of flames within spherical vessels, that are affected by curvature. In the limit for very high curvature, i.e., small flame radius, the mass diffusion and heat flux vectors may be nonaligned in respect to the unburned and burned gases velocity vectors, resulting in two-dimensional mass and heat transfer over an essentially one-dimensional flow field. The two-dimensional heat and mass transfer modifies the internal structure of the flame, altering the flame consumption speed. This type of flame alteration caused by geometrical constraints is commonly called curvature stretch. The flame stretch rate quantifies the magnitude of the flame stretch $[2,4,5,13]$

$\kappa=\frac{1}{A_{f l}} \frac{d A_{f l}}{d t}=\frac{2}{r_{f l}} \frac{d\left(r_{f l}\right)}{d t}$ 
Dowdy et al. [14] proposed a simple linear relation between the laminar flame speed and flame stretch rate,

$V_{B}=S_{L}-M_{A} \kappa$

where $S_{L}$ is the laminar flame speed, $V_{B}$ is the stretched burning velocity obtained using the Eq. (5), $M_{A}$ is the Markstein Length and $\kappa$ is the flame stretch rate. The laminar flame speed $S_{L}$ is obtained from an extrapolation of measurements of $V_{B}$ at different values of $\kappa$ to $\kappa=0$. The Markstein length is then the slope of the straight line.

\subsection{Accuracy in the Evaluation of LFS}

Considering that the evaluation of flame stretch rate has remarkably improved the results and comparison of LFS measurements, additional sources of uncertainty have been investigated during the last two decades. There are now a quite good concordance among research groups $[15,16,17]$ that the main chalenges to improve the accuracy of the results of LFS obtained in CVR's are related to: (i) mixture formation and equivalence ratio calculations (ii) mixture ignition, (iii) effects of flame confinement, (iv) flame surface instabilities, (v) flame radiation, (vi) nonlinear stretch behavior and (vii) selection of the lower and upper limits of radius for extrapolation of Eq. (7). Even though factor (i) is very important, it is directly influenced by pressure and temperatures probes, not by a termodynamic approach, and thus it will not be addressed in this work.

It is very important to match the design of the CVR with the thermodynamic method applied to post-process the data obtained in the experiments. The correct sizing of the utilized CVR in conjunction with the adequate thermodynamic approach will remarkably diminish the uncertanty related to the factors (ii) to (vii).

\section{Materials and Methods}

The objective of this section is to prepare the reportation of improvements in results of LFS obtained due to the customized design between a hardware and a software specially developed to measure LFS of outwardly premixed flames. The improvements can be grouped as: the sizing of the used CVR allowed to reduce the factors (ii) and (iii). The use of the Schlieren setup allowed to select the experiments unaffeceted by the factor (iv). The thermodynamic approach allowed to diminish the factor (v), while the conjunction use of the FORTRAN code and the sizing of the CVR allowed to reduce the factors (vi) and (vii).

\subsection{Laminar CVR Facility for Measurements of LFS}

The hardware used in this work is a spherical CVR, casted in AISI 316 stainless steel, with an inner diameter of $300 \mathrm{~mm}$ and its peak pressure rated at $350 \mathrm{bar}$. The reactor is equipped with a Kistler 6441 pressure transducer and a Schlieren Photography set up, using two opposite quartz glass windows with $150 \mathrm{~mm}$ of diameter, to provide optical access to Schlieren measurements. The mixtures are centrally spark ignited using a controlled capacitiveinductive system. A Labview based code was built to control and synchronize the spark and data acquisition. The stoichiometry of the mixtures are measured using the partial pressure method. When a liquid fuel is assessed, the gravimetric method is utilized. Hartmann [18] presented additional details on the pressure signal acquisition and treatment, the Schlieren set up and the control of the experiment. The selected Schlieren setup was the $\mathrm{Z}$ type, chosen due to its intrinsic low optical aberrations. Hartmann [18] reported an uncertainty of $0.5 \mathrm{~mm}$ in the radius calculation, when using a camera IDT model Y4-S2, set to $256 \times 256$ pixels of resolution and 10,000 fps (frames per second) of acquisition rate. Figure 2 shows a scheme of the CVR facility and the layout of the $\mathrm{Z}$ type Schlieren.

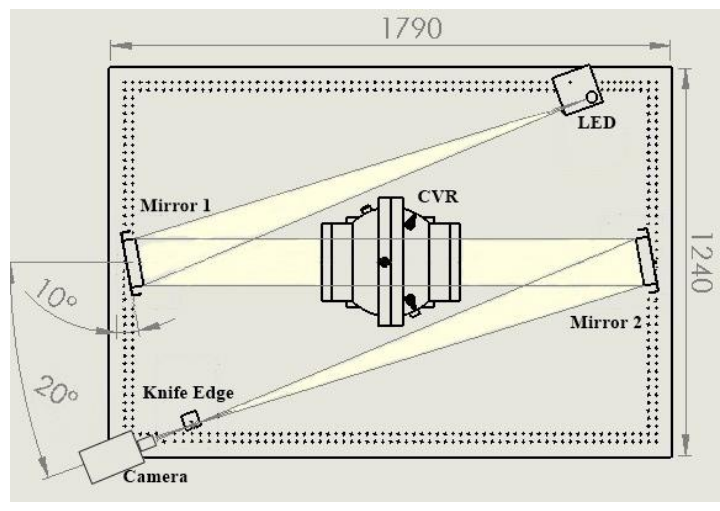

Figure 2. CVR facility and the layout of the $Z$ type Schlieren setup. Dimensions in $\mathrm{mm}$.

Since the CVR radius is $150 \mathrm{~mm}$, there is a considerable range of spatial radius to evaluate the LFS, where the pressure is about the initial pressure, an isobaric region. In this work, it were used values measured between flame radius of $40 \mathrm{~mm}$ to $60 \mathrm{~mm}$, that correspond about $25 \%$ and $40 \%$ of the reactor radius respectivelly. In this flame radius range, the flame is far from the influence of the spark, is unaffected by effects of flame confinment and is big enough to be modeled using a linear flame stretch relationship.

\subsection{Thermodynamic and Mathematical Background}

The thermodynamic model presented here to calculate the LFS using pressure trace is based on one previously developed by Rahim et al. [11], modified to avoid the need to calculate energy losses and volume corrections. The approach here is a multi-zone model, based on the following simplifying assumptions: (i) ideal gas, (ii) the flame is spherical and the flame front is smooth, (iii) the burned gases are in mechanical and chemical equilibrium and (iv) the pressure is constant within the reactor being only function of time. The multizone configuration is comprised by two main regions, a burned gas region composed by a sequence of shells burned in the previous flame steps and an unburned region that is the reactants which will be further burned by the propagation of the flame, similarly as described by Rahim et al. [11]. Between the burned and unburned region there is the flame sheet. As the flame propagates the pressure is increasing within the reactor and the burned and unburned gases are polytropic compressed,

$T_{n}=T_{\text {ref }}\left(\frac{p_{n}}{p_{\text {ref }}}\right)^{\frac{\gamma-1}{\gamma}}$

where $\gamma$ is ratio of specific heat, $T$ and $p$ are temperature and pressure, the subscript ref is the reference state and $n$ is the actual instant of the flame propagation. The vector $p_{n}=f(t)$ is the pressure trace recorded in the experiments. 
The additional thermodynamic properties are obtained using the NASA/CEA (Chemical Equilibrium with Applications), called as a subroutine of the developed FORTRAN code, that will presented in the following. The NASA/CEA code is based on ideal gas hypothesis to calculate equilibrium compostition of selected species by minimization of free energy. The set of equations is solved using the Newton-Raphson method. The NASA/CEA code is described by Gordon and McBride [19].

Knowing gases temperature, pressure and having all thermodynamic properties it is possible to evaluate the actual flame state. The flame temperature is evaluated using a feature of the NASA/CEA code, combustion process at constant pressure and enthalpy, avoiding thus the necessity to evaluate an energy equation. This approach allows to assess the flame propagation eliminating the uncertainties related to modelling heat losses.

Applying mass and volume conservation conditions and considering the burned gases are comprised of $(n-1)$ burned shells, it follows that,

$v_{i}=\sum_{j=1}^{n-1}\left(v_{j} y_{j}\right)+v_{n} y_{n}+v_{u}\left[1-y_{n}-\sum_{j=1}^{n-1}\left(y_{j}\right)\right]$

where $v$ is the specific volume, $y$ is the mass fraction of burned gases by the flame at a certain time step, $n$ is the actual time step and indicates the actual burning shell or, the flame sheet. The subscript $i$ means initial mixture conditions.

Eq. (9) is solved applying the Newton-Raphson method. The result provided by Newton-Raphson method is the burned mass fraction $y_{n}$. This sequence of calculation, i.e. isoentropic compression/constant pressure combustion/ evaluation of volume equation, is repeated until the pressure curve reaches its maximum, corresponding to the end of the flame propagation. After this main loop of calculation, the flame characteristic of interest are then obtained: the flame radius profile is calculated using Eq. (10) and the stretched burning velocity using Eq. (6).

$r_{f l}(n)=\left[\frac{3}{4 \pi}\left[\left(y_{n} v_{n}\right)+\sum_{j=1}^{n-1}\left(y_{j} v_{j}\right)\right]\right]^{1 / 3}$

The sequence of events of the main calculation loop can be summarized as follows:

1. At time step $t=1$ or $n=1$ in the Eq. (9), the first portion of fuel mixture is burned by the spark. The reactor is divided then in two regions: a very small burned gas region and an unburned gas region. The pressure is uniform and equal to $p(1)$;

2 . As the flame propagates, a new layer $n$ of the reactants is burned. This burning layer will expand, as its combustion is occurring at constant pressure $p(\mathrm{n})$ and constant enthalpy. The constant pressure combustion is based on the fact that, the characteristic combustion time has the same magnitude of the sound speed characteristic time in the unburned gases. After the end of the constant pressure combustion of the currently burning layer, the expansion wave generated by this layer travels to and reaches the reactor walls. After, the reflected pressure wave propagates back towards the reactor's center, causing the pressure to equalize throughout it, with the respective new $p(n+1)$ value. The system burned gases + burned layer + unburned gases come then to a new state of equilibrium;

3 . The step 2 is repeated until all the reactants are burned.

The use of a constant pressure combustion for each layer followed by compression and expansion of the unburned and burned regions is an acceptable simplification since the reactor is divided in small layers and each small layer burns in a small fraction of the total combustion time.

The combustion of each layer can also be described as a thermodynamic cycle. The state 1 corresponds to the unburned gases at time $t(n-1)$. A portion of this unburned gases is wrapped by the moving flame. The sequence of idealized thermodynamic processes, applied to each burning layer $n$ is summarized in Table 1 .

Table 1.Sequence of processes of an idealized flame cycle assessed by the CVR Flame Code.

\begin{tabular}{lccc}
\hline Name & Process & Source & $\begin{array}{c}\text { Properties } \\
\text { Changes }\end{array}$ \\
\hline $1 \rightarrow 2$ & Isoentropic & Previous & $T(n-1) \rightarrow \mathrm{T}(n)$ \\
& Compression & compression wave & $p(n-1) \rightarrow p(n)$ \\
\hline $2 \rightarrow 3$ & Heat addition & Burning of actual & $T_{u}(n) \rightarrow T_{b}(n)$ \\
& at constant & flame layer & $p=$ cte \\
& pressure & & \\
\hline $3 \rightarrow 4$ & Isoentropic & Actual & $T(n) \rightarrow \mathrm{T}(n+1)$ \\
& Compression & compression wave & $p(n) \rightarrow p(n+1)$ \\
\hline
\end{tabular}

It is interesting to note that in process $2 \rightarrow 3$, the burning of the actual flame layer, the change in temperature occurred at the same time step $n$. But its classification changes from unburned reactant " $u$ " to burned " $b$ ", as indicated by the respective subscripts.

In the next step of the flame propagation, the processes are repeated, considering the actual pressures $p(n)$ and $p(n+1)$ as the inputs for the next cycle.

The interpretation of the flame propagation as a sequence of three idealized processes has the advantage to offer a framework to assess energy dissipations using graphical resources. Figure 3 shows a $T-s$ diagram of the idealized CVR flame cycle.

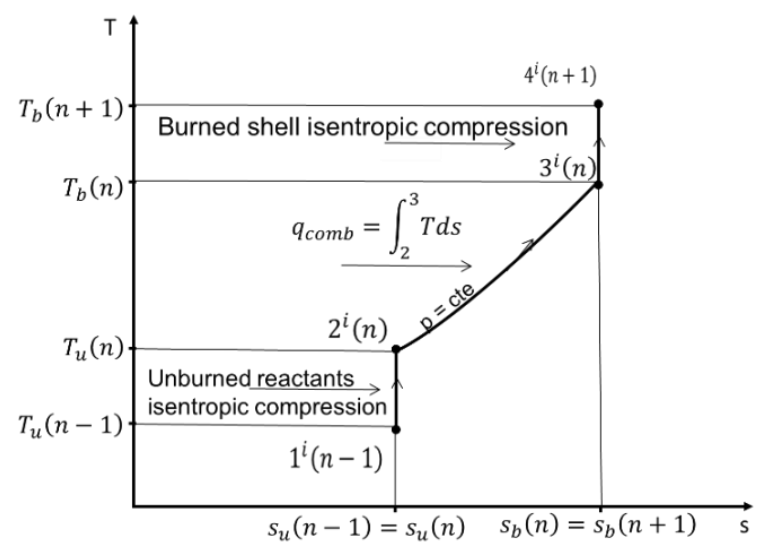

Figure 3. T-s diagram of the idealized CVR Flame Cycle. The axis are not scaled.

The real flame propagation is actually affected by heat losses. There are radiation losses from the flame to the walls and heat conduction as the flame approaches the walls. As a result of these irreversibilities, process $2 \rightarrow 3$ is 
changed, diminishing temperature of the flame sheet $T_{B}$. The process $3 \rightarrow 4$ is changed also, being not longer an isentropic compression. The key thermodynamic aspect of the Flame Code is that the main input to the calculations is the measured pressure profile. This experimental pressure is a real process pressure profile and already carries 'information' of all energy dissipations which might have occurred during the flame propagation. Thus, the comparison between the idealized process and the real pressure trace is capable to provide energy losses during the flame propagation.

Considering that the flame propagation is calculated as a sequence of constant pressure-enthalpy processes, and following the first law of thermodynamics, the heat lost at each time step $n$ is equal to the enthalpy, i.e $d q_{l}=d h$. The value of $d h$ in its turns is obtained from an equation similar to the Eq. (9),

$q_{l}(n)=d h(n)=h_{i}-\sum_{j=1}^{n-1}\left(h_{j} x_{j}\right)-h_{n} x_{n}-h_{u}\left[1-x_{n}-\sum_{j=1}^{n-1}\left(x_{j}\right)\right]$

where the enthalpy of all states of interest is taken from the NASA/CEA code.

\subsubsection{Exergetic Assessment of Premixed Spherical Flame Propagation}

The exergetic assessment applied here is based on Moran and Shapiro [20]. The exergy at a certain state is evaluated as follows,

$e=\left(h-h_{0}\right)-T_{0}\left(s-s_{0}\right)$

where $h$ is the specific enthalpy, $T$ is temperature, $s$ is the specific entropy and the subscript ' 0 ' means the dead reference state, which is defined at temperature of $298.15 \mathrm{~K}$ and pressure of $1 \mathrm{~atm}$. The chemical exergy of a certain air-fuel mixture can be calculated as follows,

$$
\begin{aligned}
& e^{c h}=\left(a h_{\text {fuel }}+b h_{O 2}+c h_{N 2}\right)^{\left(T_{i}, p_{i}\right)}-\left(d h_{C O 2}+f h_{O 2}+g h_{N 2}\right)^{\left(T_{0}, p_{0}\right)} \\
& -T_{0}\left[\left(a s_{\text {fuel }}+b s_{O 2}+c s_{N 2}\right)^{\left(T_{i}, p_{i}\right)}+\left(d s_{C O 2}+f s_{O 2}+g s_{N 2}\right)^{\left(T_{0}, p_{0}\right)}\right]
\end{aligned}
$$

where $a, b, c, d, f$ and $g$ are the reaction coefficients calculated considering complete combustion of the fuel mixture. The subscript ' $i$ ' means initial conditions. The initial exergy of a certain fuel mixture is the sum of the chemical and physical exergy, where the latter normally represents only a few percentage of the initial exergy, relevant when the initial temperature or pressure are far from the reference state.

The exergy destruction during flame propagation is mainly due to irreversibilities generated in the combustion of the reactant mixture and from the heat transferred from the hot burned gases and from the flame itself. The exergy destruction related to the burning of each discretized flame layer $n$ is,

$d e_{f l}(n)=y_{n} T_{0}\left(s-s_{0}\right)$

where $y_{n}$ is the mass fraction of burned reactants consumed by the flame at each time step $n$. The exergy destruction related to heat transfer is, $d e_{H T}(n)=\left(1-\frac{T_{0}}{T_{n}}\right) q_{l}(n)$

where $q_{l}(n)$ is the total amount of heat that crosses the control volume at the wall of the reactor, calculated at each time step $n$. using Eq. (11). Considering that the heat transfer from the burned gases and from the flame itself are the main vectors of heat loss, Beeckmann et al. [17], the flame temperature $T_{n}$ was chosen for evaluation of Eq. (15). The exergetic efficiency for the flame propagation is then calculated following [20],

$\eta_{e}=\left(\frac{e_{i}-\sum_{n_{i n i}}^{n_{f i n}} d e(n)}{e_{i}}\right)=\left(1-\frac{\sum_{n_{i n i}}^{n_{f i n}} d e(n)}{e_{i}}\right)$

where $e_{i}$ is the initial exergy of the mixture, $d e(n)$ is the sum of Eqs. (14) and (15) evaluated at each time step $n$ and $n_{i n i}$ and $n_{\text {fin }}$ are the lower and upper limits of code calculation, respectively.

\subsection{The CVR Flame Code}

The software used in this work is named CVR Flame Code. The code is better suited to the hardware described in section 3.1, but can also be adapted to additional reactors.

The code was written in FORTRAN 95 language. It is mainly intended to perform the calculations of the model described in the section 3.2 and to manage data flow with the NASA/CEA code, utilized for calculations of chemical equilibrium and thermodynamic properties. The NASA/CEA code is called to (i) provide thermodynamic properties of the gaseous mixtures, (ii) to calculate the equilibrium state of each burned shell, considering stable species and radicals involved in flame propagation and, (iii) to calculate the temperature of each flame step, considering constant enthalpy-pressure process. The equilibrium calculations number (ii) and (iii) are performed considering a more realistic mixture of burned gases, at high temperature, consisting of the following nine components: $\mathrm{CO}_{2}, \mathrm{~N}_{2}, \mathrm{H}_{2}, \mathrm{H}_{2} \mathrm{O}, \mathrm{CO}, \mathrm{NO}, \mathrm{N}_{2} \mathrm{O}, \mathrm{NO}_{2}, \mathrm{OH}$ and $\mathrm{O}_{2}$. Doing so, the results obtained using the CVR Flame Code are closer to the real processes, improving the accuracy of the results.

In a short description, the code reads the input file from experiments and print the results of interest set by the operator. The main information in the input file is the pressure trace recorded during the experiment. Figure 4 shows a pressure curve of a typical experiment., where is possible to see the lower an upper limit for calculations, $n_{\text {ini }}$ and $n_{\text {fin }}$ respectively. The both limits are assigned by the code, corresponding to the beginning of monotonic increasing of the pressure and the peak pressure value. The criteria for those selection can be set by the operator.

Figure 5 shows a plot between an input and an outcome of the CVR Flame Code, pressure as function of the calculated flame radius. It is also possible to observe the inferior and superior limits, $r_{\text {inf }}$ and $r_{\text {sup }}$ respectively, to application of the extrapolation process explained in the section 2.1.1. It is possible to see that within the interval $r_{\text {inf }}$ to $r_{\text {sup }}$ the pressure is almost the same as the initial pressure of the experiment, a region that can be considered as isobaric. 
The values of $r_{\text {inf }}$ and $r_{\text {sup }}$ can also be set by the operator, using criteria like pressure increase, linearirty of the flame stretch rate or instabilities in the flame front.

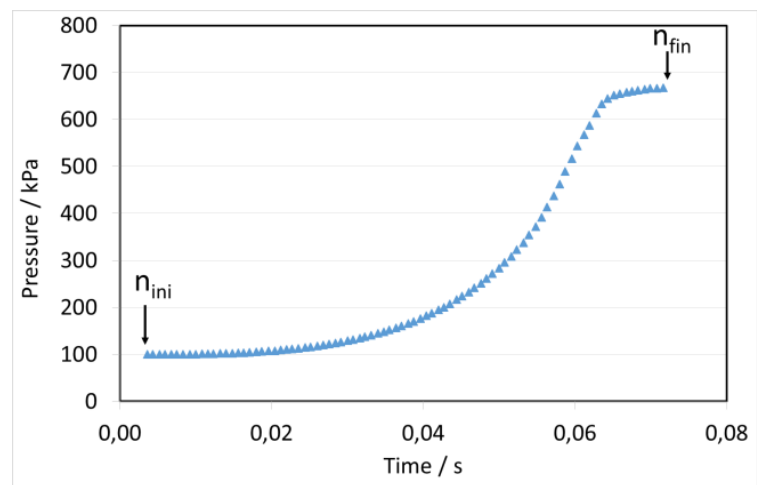

Figure 4. Experimental pressure profile, Jet A-1/air mixture, phi $=0.99$, at $408 \mathrm{~K}$ and 1 bar. The values $n_{\text {ini }}$ and $n_{\text {fin }}$ correspond to the start and end points of the code calculation, respectively.

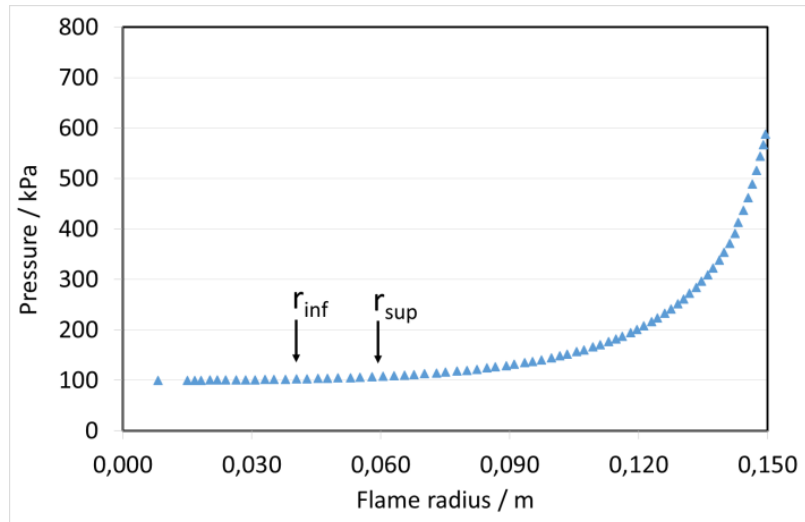

Figure 5. Experimental pressure as a funtion of calculated flame radius. Jet A-1/air mixture, phi $=0.99$, at $408 \mathrm{~K}$ and 1 bar. The limits $r_{\text {inf }}$ and $r_{\text {sup }}$ correspond to the inferior and superior radius respectively, delimiting the radius range for LFS extrapolation.

One of the mandatory factors for the obtainment of accurate results in CVR experiments using the thermodynamic approach is the quality of the pressure trace signal, where high quality signal means a smooth and monotonic curve. A monotonic pressure trace is needed because the evaluation of the volume conservation equation is sensitive to the pressure's slope, mainly in the beginning of the flame propagation when the pressure increase at each time step is very small. A smooth curve avoids oscilation in the signal of the mass fraction of the burned gases. Negative mass fraction has no physical meaning and distort the results.

The CVR Flame Code firstly reads the raw experimental data and then discretizes them. The number of discretized time steps depends on the total number of points acquired in the experiment. The CVR code applies then a sequence of two filters, the first is a simple mean filter and a second is a $5^{\text {th }}$ degree polynomial fit. The simple mean procedure is able to filter high frequency oscillations, but misses the low frequency. The polynomial curve-fit, on the other hand, is smoother and increases monotonically, causing the time derivative to vary accordingly, as the physics of the problem suggests.
After the fitting, the initial value of time considered in the calculations, called $n_{i n i}$, is selected. The selection is made to avoid any residual oscilation in the pressure slope. The value of $n_{f i n}$, the end of the flame propagation, is set to be the peak of the pressure curve. The algorithm is set to discretize the total flame propagation in about 100 steps, between $n_{i n i}$ and $n_{f i n}$. The sample size of the simple filter varies depending on this number of steps, from 5 for the faster flames to 12 for the slower flames.

The next procedure is the computation of the mixture composition and initial conditions, where the initial thermodynamic state of the mixture is evaluated, calling the CEA code. The main loop is then started: calculation of polytropic compression of the reactants, evaluation of a constant pressure-enthalpy process to obtain the flame temperature and evaluation of the specific volume equation obtaining the burned mass fraction using a NewtonRaphson algorithm. The main loop finishes when the actual burned layer $n$ is equal to $n_{\text {fin }}$. The last section of the code post-processes the burned mass fraction temporal profile, calculated in the main loop, outcoming temporal profiles of flame radius, burning velocity, flame stretch rate, exergetic assessment and linear extrapolation to get the LFS. Figure 6 depicts a flowchart of the CVR Flame Code algorithm.

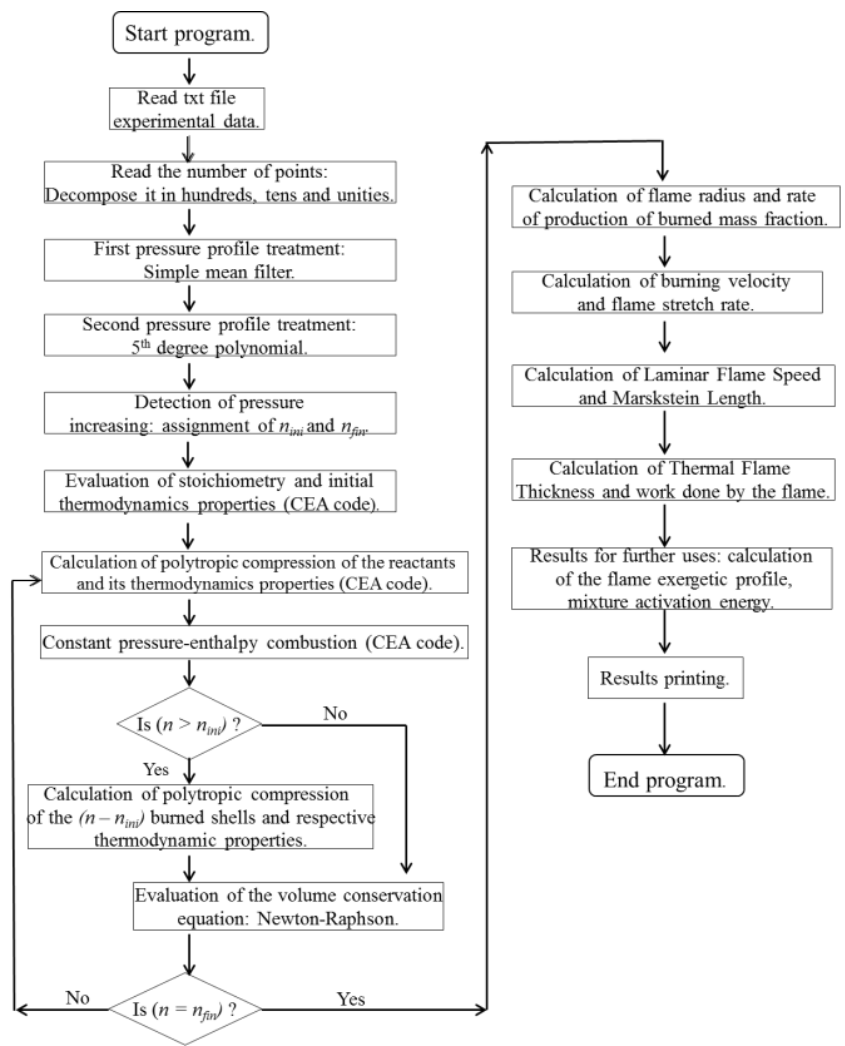

Figure 6. Flowchart of the CVR Flame Code.

Another important aspect for calculations of LFS is the extrapolation of the results plotted using Eq. (7). Figure 7 shows a plot of the calculated stretched burning velocity as a function of the flame stretch rate. Also shown are the lower radius $r_{i n f}$ and upper radius $r_{s u p}$, delimiting the socalled pre-pressure region, where the inner vessel pressure is lower than $2 \%$ of the manometric peak pressure. After the upper radius the burning velocity starts to increase due the increase in the reactants temperature, caused by polytropic compression. Lastly, the LFS can be observed as a linear extrapolation of the data at the condition of zero flame stretch rate. 


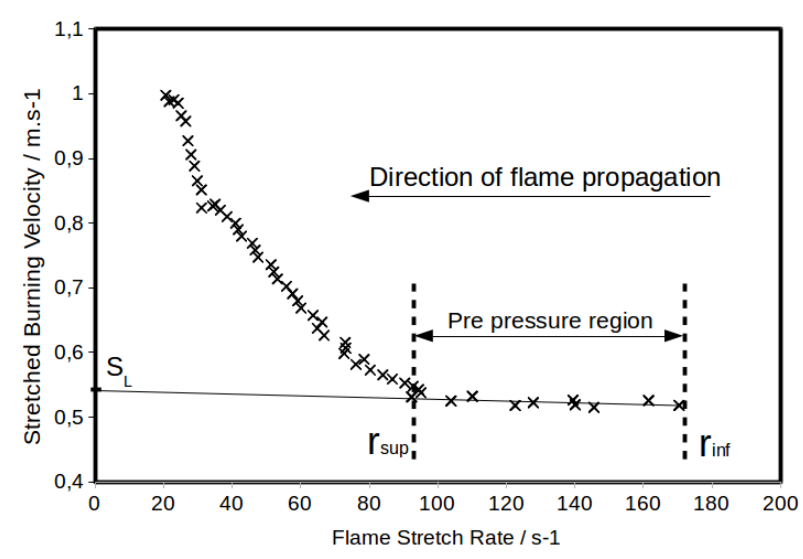

Figure 7. Stretched burning velocity of stoichiometric JetAl air mixture as function of the flame stretch rate.

As previously discussed in the beginning of section 3 , the improvements intended to minimize the uncertainties in LFS measurement were reported. In the ensuing section the results that evidence the imrpovements will be presented.

\section{CVR Flame Code Results and Comments}

The results of the CVR Flame Code were verified comparing the images from the Schlieren setup. The first procedure is to check the sphericity and smoothness of the flame, as the hypotheses (ii) listed in the section 3.1. All the reported experiments in this paper presented smooth and perfectly spherical surface, within the domain of the Schlieren measurements, as shown in Figure 8 below. When flame instabilities were visually detected in a experimental run, this experiment was discarded. Thus, the uncertainties related to the factor (iv) listed in section 2.2 were avoided.
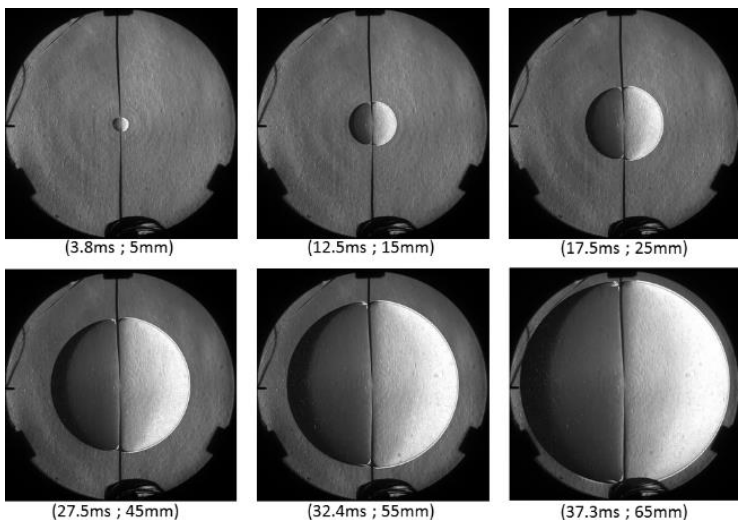

Figure 8. Sequence of Schlieren pictures of a laminar premixed flame of isooctane with air at equivalence ratio of 0.95 , initial temperature and pressure of $323 \mathrm{~K}$ and $1 \mathrm{bar}$ respectivelly. At the bottom of each picture it is shown the pair time and radius of the picture. The sequence of pictures shown a typical flame that is spherical and smooth, used in the results of LFS reported in this work.

The second comparison was made between the flame radius measured by the Schlieren method and the flame radius calculated by the CVR Flame Code. Figure 9 shows the results of the flame radius obtained using the installed Schlieren setup and the outcome of the CVR Flame Code, for a representative experiment. It is possible to see the results match very well, and the difference was always lower than $2 \%$, mostly in the beginning of the flame propagation for very small flame radius, where the values are discarded for the calculation of LFS.

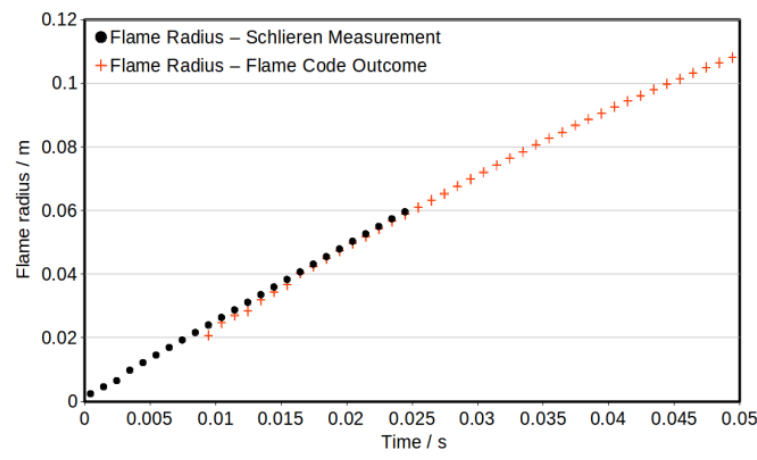

Figure 9. Flame radius CVR obtained using the Schlieren setup and the outcome of the Flame Code. The Schlieren measurements were limited by the size of the optical windows, set to $60 \mathrm{~mm}$ in this study.

\subsection{Results of Laminar Flame Speed}

Figure 10 shows results of LFS for mixtures of air and iso-octane, n-heptane, methane and Jet A-1 at initial pressure of 1 bar. It is possible to see the Jet A-1 mixtures, at $\mathrm{Ti}=408 \mathrm{~K}$, presented higher LFS for all the covered equivalence ratio interval, about $50 \%$ higher.

Figure 11 shows the calculated adiabatic flame temperature, by the constant enthalpy-pressure process used by the CVR Flame code.

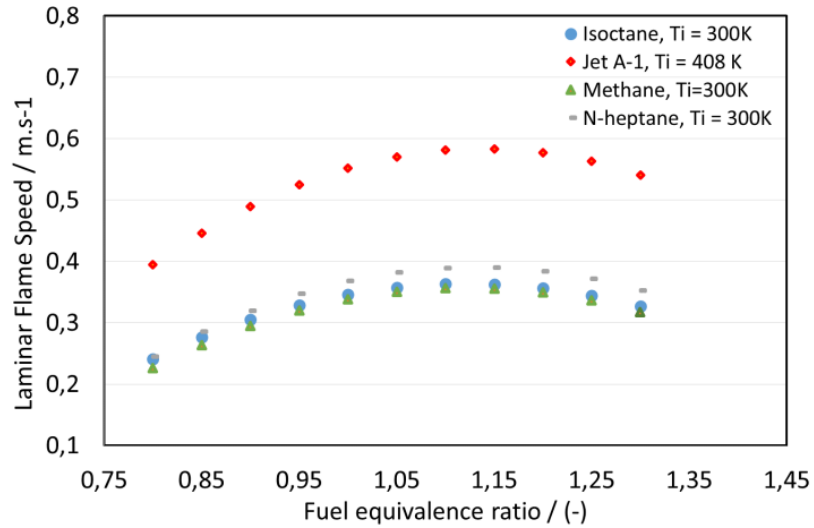

Figure 10. Laminar flame speed of mixtures of air and isooctane, n-heptane, methane and Jet A-1 at initial pressure of 1 bar, as function of the fuel equivalence ratio.

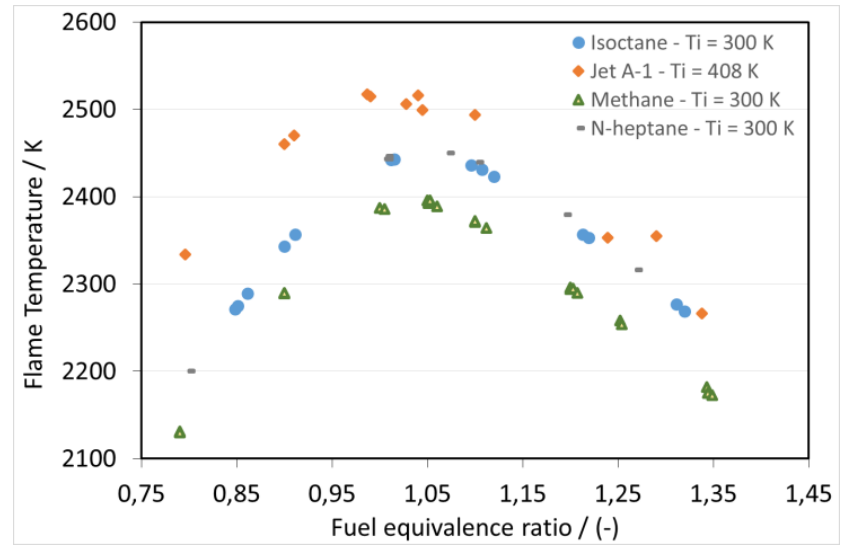

Figure 11. Calculated adiabatic flame temperature of mixtures between air and isooctane, n-heptane, methane and Jet A-1 at initial pressure of 1 bar, as function of the fuel equivalence ratio. 
It is possible to see the Jet A-1 mixtures, at $\mathrm{Ti}=408 \mathrm{~K}$, presented the higher adiabatic flame temperature, which is expected due its higher initial temperature. Additionally it shows that the LFS results showed in Figure 10 are consistent, as higher the flame temperature higher LFS will be [1-10]. Figure 12 shows the comparisons of LFS of methane/air mixtures. There are shown results adapted from Beeckmann et al. [17] obtained using a small spherical CVR, $50 \mathrm{~mm}$ inner diameter equipped with a Schlieren setup and results of simulations using the mechanism GRIMECH 3.0.

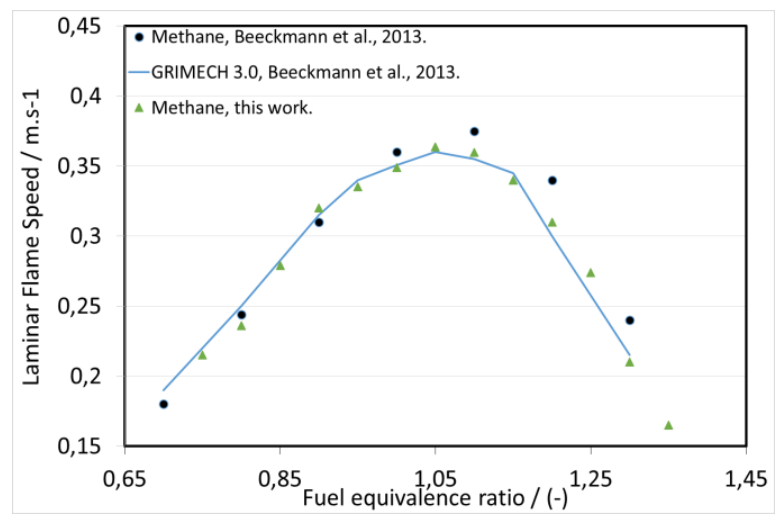

Figure 12. Laminar flame speed of mixtures of methane/air at initial temperature and pressure of $300 \mathrm{~K}$ and $1 \mathrm{bar}$, as function of the fuel equivalence ratio.

The results obtained in this work match well, mainly with the results obtained using the GRIMECH mechanism. It is also interesting to recall the experimental results presented by Beeckmann at al. were measured in a small spherical vessel, $50 \mathrm{~mm}$ diameter, where non linear effects of flame stretch play a role on the flame propagation.

\subsection{Results of Exergetic Assessment}

Figure 13 shows the exergetic efficiency of mixtures of air and isooctane, n-heptane, methane and Jet A-1 at initial pressure of 1 bar, obtained using the Eq. (16). Figure 14 shows the percentage of exergy of the initial mixture destroyed due to flame propagation processes. The points were calculated by the integration of the Eq. (14) within the interval $n_{\text {ini }}$ to $n_{\text {fin }}$. Figure 14 shows the percentage of initial mixture exergy destroyed due to heat transfer mechanisms. The points were calculated by the integration of the Eq. (15) within the internal $n_{\text {ini }}$ to $n_{\text {fin }}$.

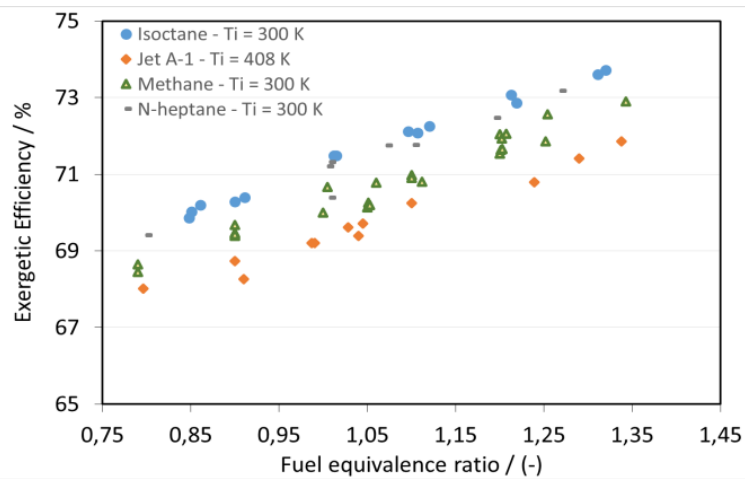

Figure 13. Exergetic efficiency of mixtures of air and isooctane, n-heptane, methane and Jet A-1 at initial pressure of 1 bar, calculated using Eq. (15), as function of the fuel equivalence ratio.

It is interesting to note the Jet A-1 mixtures presented the lowest exergetic efficiency and presented the highest
LFS. The mixtures of isooctane on the other hand, presented the highest exergetic efficiency and the lowest LFS.

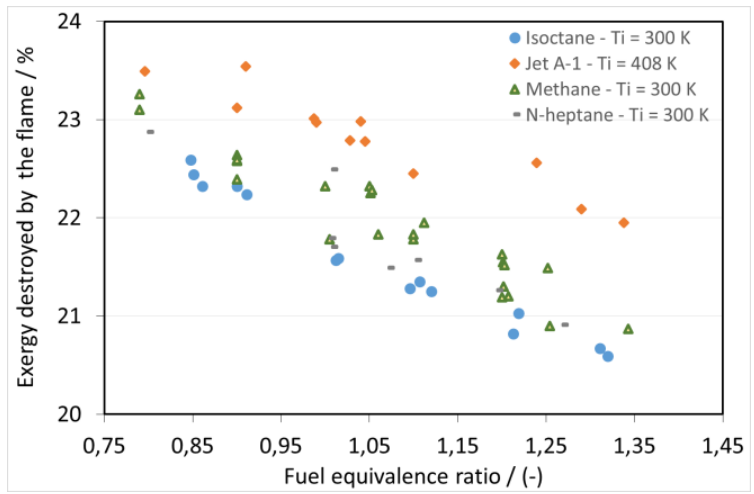

Figure 14. Percentage of initial exergy destroyed by flame propagation process of mixtures of air and iso-octane, nheptane, methane and Jet A-1 at initial pressure of 1 bar, calculated using Eq. (13), as function of the fuel equivalence ratio.

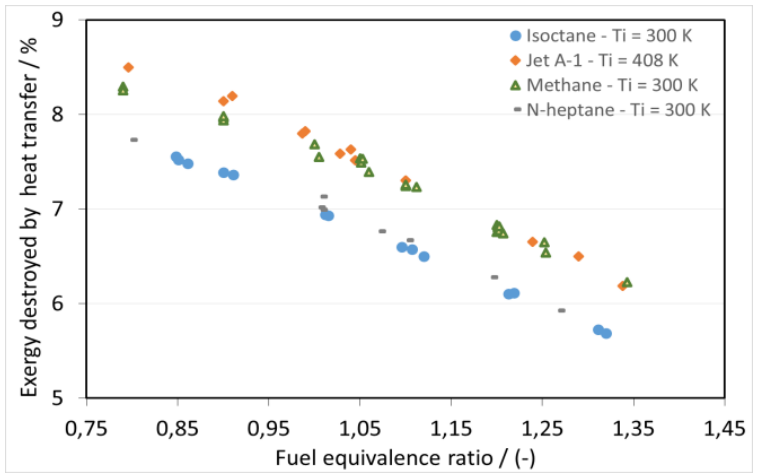

Figure 15. Percentage of initial exergy destroyed by heat transfer mechanisms of mixtures of air and iso-octane, $n$ heptane, methane and Jet A-1 at initial pressure of 1 bar, calculated using Eq. (14), as function of the fuel equivalence ratio.

Figure 14 shows the Jet A-1 mixtures presented the highest percentage of exergy destruction caused by flame propagation, calculated using Eq. (14). Considering the higher LFS is related to a higher chemical rate, and thus higher irreversibilities, this behavior was already expected. In this sense it is remarkable to note the flame exergy destruction is higher for methane compared to n-heptane and iso-octane, even though the methane mixtures presented almost the same LFS compared to n-heptane and iso-octane.

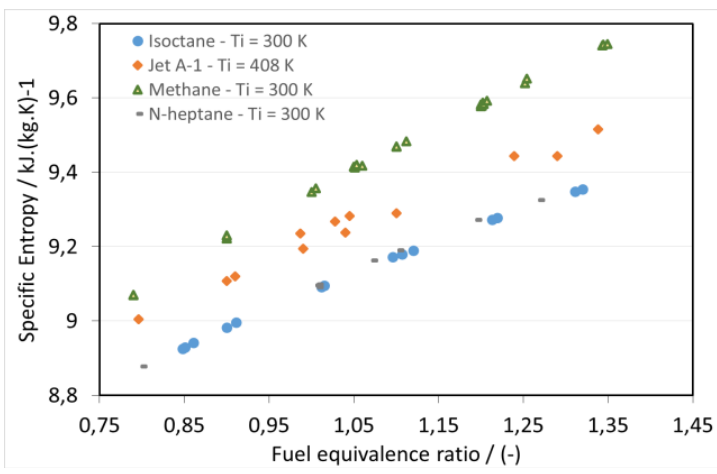

Figure 16. Calculated specific entropy of burned gases at the end of flame propagation of mixtures of air and isooctane, n-heptane, methane and Jet A-1 at initial pressure of 1 bar, calculated calling the NASA/CEA code, as function of the fuel equivalence ratio. 
Another factor that leads to high exergy destruction caused by the flame is a high specific entropy of the burned gases. Figure 16 shows the specific entropy of the burned gases at the end of the flame propagation, as function of the fuel equivalence ratio. The specific entropy was calculated calling the NASA/CEA code considering the 9 components listed in section 3.2.

It is easy to see in Figure 16 that methane mixtures presented the higher specific entropy, causing thus high percentage of exergy destruction due to flame propagation.

Figure 17 shows the volumetric fraction of water vapour in the burned gases at the end of the flame propagation, as function of the fuel equivalence ratio. Figure 18 shows the volumetric fraction of carbons dioxide, for the same mixtures showed in Figure 17. The data in Figures 17 and 18 were also obtained calling the NASA/CEA code.

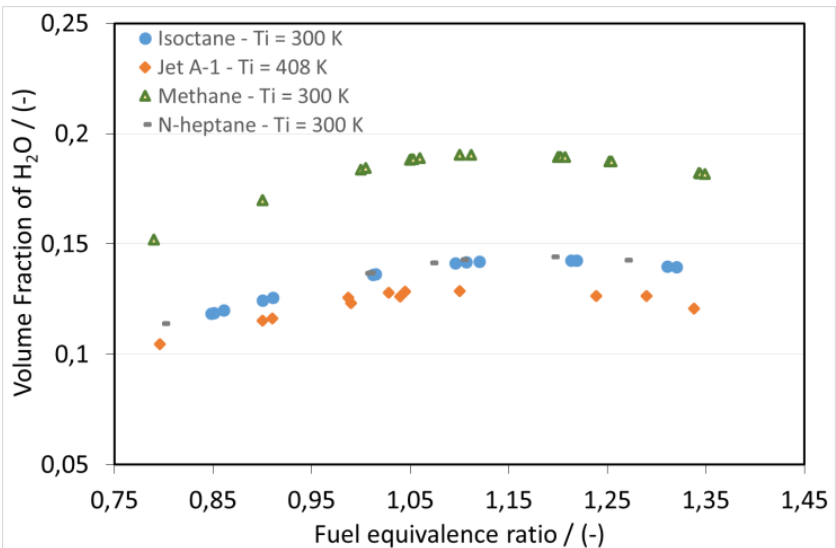

Figure 17. Calculated volumetric fraction of water vapour in the burned gases at the end of flame propagation of mixtures of air and isooctane, n-heptane, methane and Jet A-1 at initial pressure of 1 bar, calculated calling the NASA/CEA code, as function of the fuel equivalence ratio.

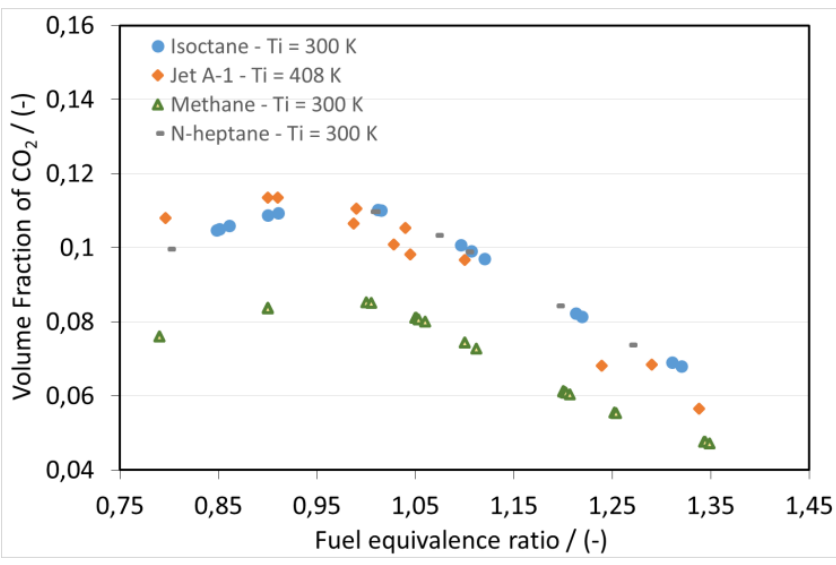

Figure 18. Calculated volumetric fraction of carbon dioxide in the burned gases at the end of flame propagation of mixtures of air and isooctane, n-heptane, methane and Jet A-1 at initial pressure of 1 bar, calculated calling the NASA/CEA code, as function of the fuel equivalence ratio.

It is possible to observe that methane mixtures presented the highest volumetric fraction of water vapour and the lowest volumetric fraction of carbon dioxide, for all the observed range of equivalence ratio. Water vapour has almost twice the specific entropy compared with carbon dioxide at the same thermodynamic state [20]. This means the results presented in Figure14 are consistent. It is worth to note that the calculated exergy destroyed by the flame is here more related to the entropy of the burned gases than to the rapidity of the flame propagation quantified by the LFS.

The second mechanism responsible for exergy destruction is related to heat transfer from the burned gases and from the flame to the reactor walls. As can be observed in Figure 15, Jet A-1 and methane mixtures presented the highest values of heat exergy destruction and the mixtures of iso-octane and n-heptane presented the lowest. It is remarkable the Jet A-1 and methane mixtures presented the same level of heat exergy destruction, notwithstanding Jet A-1 mixtures presented noticeable higher flame temperature.

Considering that the heat transfer from burned gases and from the flame occurs through radiation mechanism, obeying the Stefan-Boltzmann law, higher the temperature higher the radiation heat. It is also valid for the emissivity of the medium. Knowing the water vapour normaly has emissivity $30 \%$ higher than carbon dioxide at the same conditions [19], the comparisons of Figures 17 and 18 shows that methane flames should dissipate more heat by radiation than the others hydrocarbons investigated. Thus the results shown in Figure 15 are also consistent.

\section{Conclusions}

Results of laminar flame speed and exergetic assessment of premixed spherical flames were presented. The FORTRAN code named CVR Flame Code was presented, its main hypothesis were experimentally checked and the results of the calculated flame radius were compared with a well-known optical method, the Schlieren Fast Photography. The thermodynamic approach applied in the CVR Flame Code allows to obtain the energy losses during the flame propagation, comparing an idealized thermodynamic cycle with the real flame propagation process. The results of energy losses allowed to a comprehensive energetic and exergetic assessment of the flame propagation. The thermodynamic approach solving only the volume equation aided by the NASA/CEA Code, the CVR utilized, the selection of valid experiments using Schlieren images and the LFS evaluated in the pre-pressure region provided accurate results, avoiding the main sources of uncertainties reported so far in the literature, i.e, energy losses modelling, influence of the spark, radiation, flame surface instabilities, flame confinement, non-linear effects and extrapolation. Experiments were carried out for air mixtures with methane, iso-octane, n-heptane and Jet A-1. The results showed the laminar flame speed is the highest for the Jet fuels at higher initial temperatures and lowest for methane, about $50 \%$ for stoichiometric mixtures. The faster flames for Jet fuels presented the highest exergy destruction, about 30\%. The joint analysis of the exergy destruction mechanisms, due to heat transfer and the flame itself, showed the Jet fuel mixtures presented the highest level of exergy destruction due to heat losses and flame propagation. The auxiliary analysis of the results of adiabatic flame temperature, specific entropy of burned gases, volumetric concentration of water vapour and carbon dioxide in the burned gases showed the consistency of the exergetic assessment applied to the premixed flame propagation. The results of LFS and exergetic efficiency, aided by the auxiliary results, have shown the methodology of the CVR Flame Code is consistent and useful for exergetic assessment. Additionaly it was possible to identify and analyse diferent mechanisms of exergy 
destruction. Thus the CVR Code, in conjunction with the laminar CVR used in this work, proved to be capable to provide consitent results in studies of chemical kinetics, exergetic assessment, radiation flame heat quantification and engineering development of new fuels and its additives.

\section{Acknowledgements}

The authors thank to Petrobras - Petróleo Brasileiro S.A. for the financial support.

\begin{tabular}{|c|c|}
\hline \multicolumn{2}{|c|}{ Acronyms } \\
\hline$C V R$ & Constant Volume Reactor \\
\hline$d e$ & Exergy destroyed \\
\hline$L F S$ & Laminar Flame Speed \\
\hline \multicolumn{2}{|c|}{ Nomenclature } \\
\hline$A$ & Area, $\mathrm{m}^{2}$ \\
\hline$e$ & Specific exergy, $\mathrm{kJ} / \mathrm{kg}$ \\
\hline$h$ & Specific enthalpy, $\mathrm{kJ} / \mathrm{kg}$ \\
\hline$m$ & Mass, kg \\
\hline$n$ & Discretized time step, non-dimensional \\
\hline$p$ & Pressure, $\mathrm{kPa}$ \\
\hline$r$ & Radius, m \\
\hline$s$ & Specific entropy, kJ/kg-K \\
\hline$t$ & Time, s \\
\hline$T$ & Temperature, $\mathrm{K}$ \\
\hline$v$ & Specific volume, $\mathrm{m}^{3} / \mathrm{kg}$ \\
\hline$V$ & Burning velocity, $\mathrm{m} / \mathrm{s}$ \\
\hline$V$ & Volume, $\mathrm{m}^{3}$ \\
\hline$y$ & mass fraction , $\mathrm{kg} / \mathrm{kg}$ \\
\hline \multicolumn{2}{|c|}{ Greek Symbols } \\
\hline$\kappa$ & Flame stretch rate, $1 / \mathrm{s}$ \\
\hline$\rho$ & Density, $\mathrm{kg} / \mathrm{m}^{3}$ \\
\hline \multicolumn{2}{|c|}{ Subscripts and superscripts } \\
\hline$b$ & Burned \\
\hline$c h$ & Chemical \\
\hline$B L$ & Boundary Layer \\
\hline $\mathrm{C}$ & Chamber \\
\hline$f$ & Final \\
\hline$f l$ & Flame \\
\hline$H L$ & Heat Losses \\
\hline$i$ & Initial \\
\hline$l s$ & Lost \\
\hline$u$ & Unburned \\
\hline 0 & Reference state \\
\hline
\end{tabular}

\section{References:}

[1]F. A. Williams, Combustion Theory. $1^{\text {st }}$ Ed., Boston/USA: Perseu Books, 1986.

[2] I. Glassman, \& R. A. Yetter, Combustion. $4^{\text {th }}$ Ed., San Diego/USA: Elsevier Inc., 2008.

[3] J. Warnatz, U. Maas, \& R. W. Dibble, Combustion Physical and Chemical Fundamentals, Modeling and Simulation, Experiments, Pollutant Formation. $4^{\text {th }}$ Ed., New York/USA: Springer 2006.

[4] T. Poinsot, \& D. Veynante, Theoretical and Numerical Combustion. $2^{\text {nd }}$ Ed., Philadelphia/USA: Edwards, 2005.

[5] C. K. Law, \& C. K. Wu, "On the determination of laminar flame speeds from stretched flames." in Twentieth Symposium (International) on Combustion/The Combustion Institute, (pp. 1941-1949), 1984.

[6] C. K. Law, "Dryden Lecture: Fuel Options for Next Generation Chemical Propulsion." Proceedings of $49^{\text {th }}$ AIAA Aerospace Science Meeting including the New
Horizons Forum And Aerospace Exposition, Orlando/USA, 1-30, 2011.

[7] G. S. Setles, Schlieren and Shadowgraph Techniques. $1^{\text {st }} E d$., New York/USA: Springer, 2001.

[8] B. Hopkinson, "Explosions of Coal-Gas and Air," Proceedings of the Royal Society of London, 77(518), 387-413, 1906.

[9] M. G. Zabetakis, Flammability characteristics of combustible gases and vapors, $1^{\text {st }} \mathrm{Ed}$, Washington D.C./USA: US Dpt. of Interiror, Bureau of Mines, 1965.

[10] M. Metghalchi, \& J. C. Keck, "Burning Velocities of Mixtures of Air with Methanol, Isooctane, and Indolene at High Pressure and Temperature," Combustion and Flame, 48, 191 - 210, 1982.

[11] F. Rahim, K. Eisazadeh-Far, F. Parsinejad, R. J. Andrews, \& H. Metghalchi, "A thermodynamical model to calculate burning speed of methne-air-diluent mixtures," Int. J. of Thermodynamics, 11, 151-160, 2008.

[12] M. Matalon, "Intrinsic Flame Instabilities in Premixed and Nonpremixed Combustion," Annu. Rev. Fluid Mech, 39, 163-91, 2007.

[13] C. K. Law, \& C. J. Sung, "Structure, aerodynamics, and geometry of premixed flamelets," Progress in Energy and Combustion Science, 26, 459-505, 2000.

[14] D. R. Dowdy, D. B. Smith, S. C. Taylor, A. Williams, "The use of expanding spherical flames to determine burning velocities and stretch effects in hydrogen/air mixtures," in Twenty-Third Symposium (International) on Combustion/The Combustion Institute, (pp. 325332), 1991.

[15] F. N. Egolfopoulos, N. Hansen, Y. Ju, K. KohseHöinghaus, C. K. Law, F. Qi, “Advances and challenges in laminar flame experiments and implications for combustion chemistry," Progress in Energy and Combustion Science, 43, 36-67, 2014.

[16] Z. Chen, "On the accuracy of laminar flame speeds measured from outwardly propagating spherical flames," Combustion and Flame, 2442-2453, 2015.

[17] J. Beeckmann, N. Chaumeix, P. Dagaut, G. Dayma, F. G. Egolfopoulos, F. Foucher, "Colaborative Study for Accurate Measurements of Laminar Burning Velocity," Proceedings of the European Combustion Meeting, 1-6, 2013.

[18] Hartmann, E. M. (2014). Instrumentação e Operacionalização de um Reator de Volume Constante para Medição de Velocidade de Chama (Master dissertation), UFSC, Florianópolis, Brazil.

[19] S. Gordon, and B. J. McBride, "Computer Program for Calculation of Complex Chemical Equilibrium Compositions and Applications." NASA Technical Report, Reference Publication 1311, Cleveland/USA, 1994.

[20] M. J. Moran, \& H. N. Shapiro, Fundamentals of Engineering Thermodynamics. 2nd Ed., New York/USA: John Wiley and Sons Inc., 2004. 